TRABAJOS ORIGINALES

Rev Obstet Ginecol Venez. 2020; 80 (4): 268-279

https://doi.org/10.51288/00800404

\title{
Desempeño del índice proteína/creatinina para predecir proteinuria significativa en pacientes con sospecha de preeclampsia
}

\author{
Drs. Johana Karin Santa María-Ortiz¹, Mariela Zavaleta León ${ }^{1}$.
}

\begin{abstract}
RESUMEN
Objetivo: Evaluar el desempeño del indice proteina/creatinina para predecir proteinuria significativa $y$ secundariamente, hallar el mejor punto de corte que tuviera mejor sensibilidad y especificidad.

Métodos: Estudio de prueba diagnóstica, desarrollado en un diseño transversal de pacientes expuestas al síndrome hipertensivo del embarazo las cuales desarrollaron o no preeclampsia posterior. Se seleccionaron de forma no probabilística a 173 pacientes. Se halló el nuevo punto de corte del índice proteína/creatinina mediante la curva ROC. Se calculó su sensibilidad, especificidad, valor de predicción positivo y negativo, likelihood ratios o razones de verosimilitud positiva y negativa y se relacionó con la proteinuria de 24 horas mediante la correlación de Spearman. El análisis de datos se realizó con el software STATA, versión 13.

Resultados: El nuevo de punto de corte fue 0,39 con una sensibilidad de 68,3\%, especificidad de 73,5\%, valor de predicción positivo 91,3\%, valor de predicción negativo de 36,2\%, likelihood ratio + de 2,58, likelihood ratio - de 0,43 y un área bajo la curva de 0,7799. Se obtuvo una excelente correlación con la proteinuria de 24 horas, $(r s=0,9308, p=0,000)$.

Conclusión: Si bien el nuevo punto de corte para el índice proteína/creatinina es diferente al convencional, este es más especifico, por lo que podría ser más útil en el manejo ambulatorio de las pacientes de una población con baja sospecha de preeclampsia.
\end{abstract}

Palabras clave: Preeclampsia, Índice Proteina/Creatinina, Proteinuria de 24 horas.

\section{SUMMARY}

Objective: To assess the performance of the protein/creatinine index to predict proteinuria significantly and secondarily, to find the best cut-off point that had better sensitivity and specificity.

Methods: Diagnostic test study developed in a cross-sectional design of patients exposed to the hypertensive syndrome of pregnancy who did or did not develop posterior preeclampsia. 173 patients were selected non-probabilistically. The new cutoff of protein/creatinine index was found using the ROC curve, we calculated sensitivity, specificity, positive and negative prediction value, positive and negative likelihood ratios and find the Spearman correlation with 24 hours urine proteinuria. Data analysis was performed using STATA software version 13.

Results: The new cutoff was 0.39 with $68.3 \%$ sensitivity, specificity $73.5 \%$, positive prediction value $91.3 \%$, negative prediction value $36.2 \%$, likelihood ratios (+) 2.58, likelihood ratios (-) 0.43 and an area under the curve of 0.7799 . Excellent correlation was obtained with 24 hours proteinuria ( $r s=0.9308, p=0.000)$.

Conclusion: Although the new cutoff for the protein/creatinine index is different from conventional, this conventional this is more specific and therefore may be useful in the outpatient management on patients with low suspicious of preeclampsia in our population.

Keywords: Preeclampsia, Protein/Creatinine index, 24 hours proteinuria.

\section{INTRODUCCIÓN}

La preeclampsia es la patología médica más importante en términos de morbilidad y mortalidad materno perinatal $(1,2)$. En el Perú, ha demostrado ser la segunda causa de muerte materna $(17 \%$ al

${ }^{1}$ Médico Cirujano, Servicio de Ginecología y Obstetricia, Hospital Cayetano Heredia, Lima-Perú.
$21 \%)(3,4)$. El diagnóstico de preeclampsia, según 


\section{DESEMPEÑO DEL ÍNDICE PROTEÍNA/CREATININA PARA PREDECIR PROTEINURIA SIGNIFICATIVA EN PACIENTES CON SOSPECHA DE PREECLAMPSIA}

la última guía del Colegio Americano de Obstetricia y Ginecología (ACOG), publicada en noviembre del 2013 (5), se determina por la presencia de presión arterial sistólica $(\mathrm{PAS}) \geq 140 \mathrm{~mm} \mathrm{Hg}$ y/o presión arterial diastólica $(\mathrm{PAD}) \geq 90 \mathrm{~mm} \mathrm{Hg}$, medida en 2 oportunidades con un intervalo mínimo de 4 horas, después de la semana 20 de gestación, en una paciente previamente normotensa; asociada a una proteinuria igual o mayor a $300 \mathrm{mg} / \mathrm{dl}$ en 24 horas o un índice de proteína/creatinina $(\mathrm{I} / \mathrm{C}) \geq 0,3$. El diagnóstico de preeclampsia con signos de gravedad requiere además de la presencia de hipertensión arterial, el hallazgo de trombocitopenia menor < a 100000 plaquetas/ microlitro, insuficiencia renal (creatinina $>1,1 \mathrm{mg} / \mathrm{dl}$ o su duplicación), disfunción hepática (duplicación de transaminasas respecto a valor basal), edema pulmonar y/o la presencia de síntomas visuales o cerebrales (5).

Hasta hace unos años, la cuantificación de proteínas en orina de 24 horas se consideraba el estándar de oro para la medición de proteinuria en gestantes con sospecha de preeclampsia $(6,7)$. A pesar de ello, se han documentado desventajas importantes, como el tiempo que demanda su recolección y conservación, generando una mayor estancia hospitalaria y una recolección incompleta en más del $50 \%$ de las pacientes. Esto hace que no esté disponible para decisiones clínicas en una primera evaluación, retrasando el diagnóstico y generando una mayor incidencia de complicaciones maternoperinatales (8 11). Debido a estas desventajas se ha propuesto como método alternativo el índice proteína/creatinina (5). En los últimos años, se considera una prueba rápida y fácil de aplicar para la detección de proteinuria significativa (12 - 19). En algunos estudios, ha sido probado como un buen sustituto de la proteinuria de 24 horas en pacientes con enfermedades renales como nefropatía diabética, nefritis lúpica, enfermedad renal crónica y casos de trasplante renal, demostrándose una buena correlación entre los dos métodos (20 23). Sin embargo en muchos países de bajos recursos, la tira reactiva sigue siendo de uso rutinario para el diagnóstico temprano de proteinuria significativa en pacientes con sospecha de preeclampsia, a pesar de su baja sensibilidad y especificidad (24). El ACOG solo aprueba su uso en caso de no contar con la cuantificación de proteinuria de 24 horas o el hallazgo del índice proteína/creatinina (5).

El uso rutinario del índice proteína/creatinina sobre la recolección de la proteinuria de 24 horas, tiene ventajas significativas, que incluyen una pronta toma de decisiones clínicas, disminución de costos y recursos de salud, y menor tiempo de hospitalización $(12-19)$. Se han registrado diversos puntos de corte del índice proteína/creatinina para la determinación de proteinuria significativa en relación con la proteinuria de 24 horas, con un rango de variación de 0,15 a 0,50. Este amplio intervalo podría deberse a características demográficas, antropométricas y/o el estado nutricional de cada población (13).

En Perú, no existen estudios en pacientes con sospecha de preeclampsia que sugieran un punto de corte para esta prueba diagnóstica. Es importante validar el punto de corte propuesto por el Colegio Americano de Obstetricia y Ginecología, ya que este podría variar, dadas las diferentes características demográficas y antropométricas antes mencionadas, que presenta cada tipo de población y que probablemente dista de la población americana. Por tanto, resulta importante definir un punto de corte para cada población y describir la variabilidad que presenta respecto a lo sugerido recientemente por el ACOG.

El objetivo primario del estudio fue evaluar el desempeño del índice proteína/creatinina para predecir proteinuria significativa y secundariamente, hallar el mejor punto de corte que tuviera mejor sensibilidad y especificidad. 


\section{MÉTODOS}

Se trata de un estudio de prueba diagnóstica desarrollado en un diseño transversal de pacientes expuestas al síndrome hipertensivo del embarazo las cuales desarrollaron o no preeclampsia posterior. Las pacientes fueron seleccionadas en los ambientes de emergencia y consultorio externo del Departamento de Obstetricia y Ginecología del Hospital Cayetano Heredia en Lima - Perú, mediante un método de muestreo por conveniencia y no probabilístico, entre los meses de enero y diciembre de 2019.

La población fueron pacientes embarazadas con hipertensión gestacional a descartar preeclampsia; se excluyeron pacientes con retraso mental, infección urinaria, lupus, diabetes mellitus o gestacional, enfermedad renal, tuberculosis, virus de inmunodeficiencia adquirida (VIH) y uropatías.

Test índice: relación proteína creatinina (punto de corte 0,3$)$.

Test de referencia estándar: proteinuria significativa por recolección de 24 horas (punto de corte $300 \mathrm{mg}$ / dL).

Valoración de los test en términos de: sensibilidad, especificidad, valor predictivo positivo, valor predictivo negativo, área bajo la curva.

La sospecha de preeclampsia se planteó en toda gestante con presión arterial sistólica $\geq 140 \mathrm{mmHg}$ y/o presión arterial diastólica $\geq 90 \mathrm{mmHg}$, en dos oportunidades con un intervalo mínimo de 4 horas entre las tomas, en una paciente en reposo. Para determinar la gravedad del cuadro se solicitaron exámenes de laboratorio: plaquetas, creatinina sérica, transaminasas, deshidrogenasa láctica, bilirrubina, urea y hematocrito. Al momento del ingreso se tomó una alícuota de 5 a $10 \mathrm{ml}$ de una muestra de orina de chorro medio, en la que se cuantificó la concentración de proteinuria (reactivo de pirogalol) y creatinuria (método de Jaffe enzimático) con la técnica de química seca estándar del laboratorio del Hospital Cayetano Heredia; con estos resultados se halló el cociente proteína/creatinina tomando como referencia el punto de corte estándar $(0,3)$. Posteriormente se procedió a la recolección de orina en 24 horas, el test estándar de oro, donde se halló la proteinuria de 24 horas (punto de corte $300 \mathrm{mg} / \mathrm{dL}$ ) (ensayo turbidimétrico con reactivo de tricloro de ácido acético). Durante la hospitalización se monitorizó la presión arterial materna y la evolución de signos y síntomas como: cefalea, escotomas, fotopsias, epigastralgia, oliguria, náuseas y vómitos. Se realizó un seguimiento estricto de las pacientes registrando las complicaciones maternofetales y su evolución hasta el alta médica. Con toda la información se procedió al llenado de una ficha de recolección de datos y la creación de una base de datos en formato Excell, especialmente diseñadas para el estudio, donde se registró antecedentes importantes, cuadro clínico, resultados de laboratorio y evolución.

Se procedió a la evaluación estadística de las características generales de la población de estudio. Las variables categóricas se compararon con la prueba de chi cuadrado y las variables continuas mediante la t-Student y suma de rangos de Wilcoxon, dependiendo de si la variable tiene distribución normal o no, lo que se halló mediante la prueba de Shapiro Wilk. Para comparar más de dos medias se usó ANOVA y ONEWAY y Kruskal Wallis, dependiendo de la distribución de la variable. Se estratificó el índice proteina/creatinina y la proteinuria de 24 horas según sus puntos de corte convencionales $(\geq 0,3 \mathrm{y} \geq 300$ $\mathrm{mg}$ /día) y se halló la sensibilidad ( $\mathrm{S}$ ), especificidad (E), valor de predicción negativo (VPN), valor de predicción positivo (VPP), likelihood ratios o razones de verosimilitud positivo $(\mathrm{LHR}+) \mathrm{y}$ negativo (LHR-). Se evaluó la correlación entre el índice proteína/creatinina y la proteinuria de 24 horas utilizando un gráfico de correlación de dos vías y el 
coeficiente de correlación de Spearman (rs). Se diseñó la curva ROC para la proteinuria de 24 horas y el índice proteína/creatinina, con la cual, para el último, se halló el mejor punto de corte para la población en estudio y se calculó sus nuevos valores de S, E, VPN, VPP, LHR (+) y LHR (-). Finalmente, se compararon las áreas bajo las curvas de ambas pruebas utilizando el test de igualdad de dos curvas ROC. Los datos fueron analizados con el software STATA versión 13. Para todos los resultados se consideró un $\mathrm{p}$ estadísticamente significativo $\leq 0,05$.

Todas las pacientes firmaron el consentimiento informado y el estudio fue aprobado por el comité de ética de la Facultad de Medicina de la Universidad Peruana Cayetano Heredia y del Hospital Cayetano Heredia.

\section{RESULTADOS}

Durante el periodo de enero a diciembre de 2019, se registraron 4367 ingresos a la Unidad de Hospitalización del Departamento de Obstetricia y Ginecología del Hospital Cayetano Heredia, 348 pacientes $(7,9 \%)$ tuvieron como diagnóstico inicial síndrome hipertensivo del embarazo a descartar preeclampsia. Para el estudio se seleccionaron 173 pacientes, cuyas características generales se muestran en la tabla 1 .

La mediana de la edad fue 27 años (RI 22-33,5). La mediana de la edad gestacional fue 37,6 semanas (RI 35,25 - 39,1). Esta se calculó a partir de una FUR confiable en 113 pacientes ( $66 \%$ ) y en 27 pacientes $(15,6 \%)$ se calculó a partir de la ecografía del primer trimestre, 33 pacientes (19\%) no contaban con esta información. El número de gestaciones previas osciló entre 0 y 9 , siendo 59 pacientes $(34,3 \%)$ primigestas. El rango de paridad fue de 0 a 7 y 78 (45,35 \%) eran nulíparas. Los antecedentes más importantes fueron: cesárea anterior en 47 pacientes $(27,1 \%)$, preeclampsia en gestación
Tabla 1. Características generales de las pacientes.

\begin{tabular}{lcc}
\hline Variable & Mediana & $\begin{array}{c}\text { Rango } \\
\text { intercuartílico (RI) }\end{array}$ \\
\hline Edad & 27 & $22-33,5$ \\
Edad gestacional & 37,6 & $35,25-39,1$ \\
Peso materno & & \\
$\quad$ Peso habitual & 63 & $55-72$ \\
$\quad$ Peso final gestación & 75 & $66-83$ \\
$\quad$ Variación de peso & 10,5 & $7-15$ \\
Paridad & $\mathrm{n}$ & $\%$ \\
$\quad$ Nulíparas & 78 & 45,35 \\
$\quad$ Primíparas & 42 & 24,42 \\
$\quad$ Paridad $\geq 2$ & 52 & 30,23 \\
Gestación & & \\
$\quad$ Primigesta & 59 & 34,3 \\
$\quad$ Segundigesta & 44 & 25,58 \\
$\quad$ Tercigesta & 30 & 17,44 \\
$\quad$ Multigesta $(\geq 4)$ & 39 & 22,68 \\
Control prenatal & & \\
$\quad<6$ consultas & 70 & 40,46 \\
$\quad \geq 6$ consultas & 103 & 59,54 \\
\hline
\end{tabular}

previa en $32(19,5 \%)$ e hipertensión crónica en 6 $(3,4 \%)$.

Cincuenta y un pacientes $(21,4 \%)$ fueron asintomáticas al ingreso. La tabla 2 muestra que los signos y síntomas más frecuentes fueron: cefalea en 91 casos $(52,6 \%)$, edema en miembros inferiores en 77 (44,5\%), tinnitus en $33(19 \%)$ y epigastralgia en $25(14,45 \%)$. La mediana de la presión arterial sistólica fue $140 \mathrm{~mm} \mathrm{Hg}$ (RI 135-150) y de la presión arterial diastólica $90 \mathrm{~mm}$ Hg (RI 90-95).

Los resultados de laboratorio se categorizaron según los puntos de corte definidos como criterios de gravedad para preeclampsia y síndrome HELLP. Cuatro pacientes $(2,31 \%)$ presentaron menos de 100000 plaquetas/microlitros, 25 (14,45\%) tuvieron elevación de los niveles de transaminasa glutámico 
Tabla 2. Signos y síntomas.

\begin{tabular}{lcc}
\hline Variable & $\mathrm{n}$ & $\%$ \\
\hline Cefalea & 91 & 52,5 \\
Edemas en miembros inferiores & 77 & 44,5 \\
Tinnitus & 33 & 19 \\
Edema miembros superiores & 26 & 15 \\
Epigastralgia & 25 & 14,4 \\
Escotomas & 23 & 13,29 \\
Naúseas & 9 & 5,2 \\
Vómitos & 7 & 4 \\
Fotopsias & 7 & 4 \\
Hiperreflexia & 6 & 3,4 \\
Sangrado & 2 & 1,16 \\
\hline
\end{tabular}

pirúvica (TGP), 14 (8,09\%) de transaminasa glutámico oxalacética (TGO), 69 (39,88\%) de deshidrogenasa láctica (DHL), 4 (4,3\%) de bilirrubina y en 4 casos $(2,37 \%)$ la creatinina sérica fue igual o mayor a $1,2 \mathrm{mg} / \mathrm{dL}$ (Tabla 3 ).

Tabla 3. Exámenes de laboratorio

\begin{tabular}{lcc}
\hline Variable & Mediana & RI \\
\hline Hematocrito (\%) & 34 & $33-37$ \\
Plaquetas/microlitros & 250 & $196-299$ \\
$\begin{array}{l}\text { Bilirrubina mg/dl } \\
\text { Transaminasa glutámico } \\
\text { oxalacética mg/dl }\end{array}$ & 0,4 & $0,3-0,6$ \\
$\begin{array}{l}\text { Transaminasa glutámico } \\
\text { pirúvica mg/dl }\end{array}$ & 28 & $20-39$ \\
$\begin{array}{l}\text { Deshidrogenasa láctica mg/dl } \\
\text { Urea mg/dl }\end{array}$ & 515 & $445-654$ \\
Creatinina mg/dl & 20 & $15,6-24$ \\
\hline
\end{tabular}

Durante la evolución de cada paciente se registró el flujo urinario en 24 horas, el menor valor registrado tuvo una mediana de $0,8 \mathrm{cc} / \mathrm{h}$ (RI 0,6-1,04). Treinta y cuatro pacientes $(20,61 \%)$ presentaron oliguria.

De las 173 pacientes incluidas en el estudio, 155 culminaron la gestación durante su hospitalización. En 18 casos (10\%) no se registró la indicación de término de la gestación. El parto abdominal fue el más frecuente $(76,76 \%)$.

Entre las complicaciones maternas se observó 3 pacientes $(1,9 \%)$ con síndrome HELLP, 2 (1,29\%) con atonía uterina y $1(0,6 \%)$ con eclampsia.

La mediana de la edad gestacional de los recién nacidos calculada por Capurro fue 38 semanas (RI 37-39), la talla $50 \mathrm{~cm}$ (RI 47,5-51) y el perímetro cefálico $34 \mathrm{~cm}$ (RI 33-35). La mediana del peso fue 3090 gramos (RI 2625 - 3425), 27 recién nacidos (17,41\%) presentaron bajo peso, 3 (1,9\%) muy bajo peso y 3 (1,9\%) extremo bajo peso. Las complicaciones fetales y/o neonatales fueron: prematuridad en 37 casos $(23,8 \%)$, restricción de crecimiento intrauterino (RCIU) en 19 (12,2\%), oligohidramnios en 3 (1,9\%), sufrimiento fetal agudo en $6(3,8 \%)$ y óbito fetal en $3(1,9 \%)$.

Según los criterios recientemente estandarizados por el Colegio Americano de Obstetricia y Ginecología para el diagnóstico de preeclampsia (5), se encontró que 95 pacientes $(54,91 \%)$ presentaron preeclampsia leve, $44(25,43 \%)$ preeclampsia grave y en 34 $(19,65 \%)$ se descartó la misma.

La mediana del índice proteína/creatinina fue de 0,55 (RI 0,29-1,32). Según el punto de corte convencional, 128 pacientes (74 \%) presentaron un índice igual o mayor a 0,3 , de ellas $115(89,8 \%)$ tuvieron como diagnóstico final preeclampsia. Las propiedades discriminativas diagnósticas de este punto de corte fueron: S 82,7 \% (IC $95 \%$ : 75,6-88,1), E 61,8 \% (IC 
$95 \%$ : 45-76,1), VPP 89,8 \% (IC $95 \%$ : 83,4-94), VPN 46,7 \% (IC $95 \%: 32,9-60,9)$, LHR (+) 2,16 (IC $95 \%$ : 1,4-3,34), LHR (-) 0,28 (IC 95 \%: 0,19-0,41).

De igual manera, se calculó la mediana para la proteinuria de 24 horas, la cual resultó ser 483,5 mg/d (RI 262,8 - 1110). Según el punto de corte convencional ( $\geq 300 \mathrm{mg} / \mathrm{d}), 123$ pacientes $(71,1 \%)$ presentaron proteinuria significativa, de ellas $113(91,9 \%)$ tuvieron preeclampsia. Se obtuvo una excelente correlación de 0,9308 entre el índice proteína/creatinina y la proteinuria de 24 horas $(\mathrm{p}<0,000)$ (Gráfico 1).

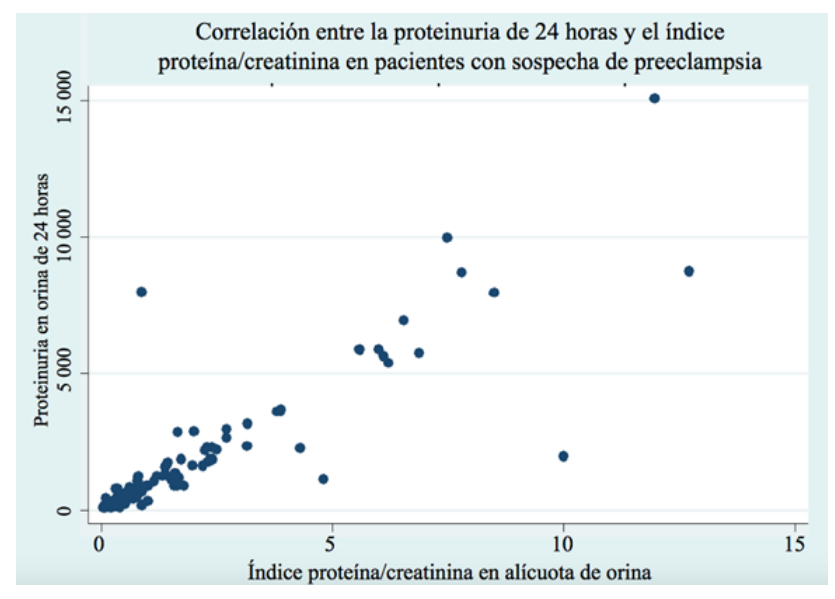

Gráfico 1. Correlación entre la proteinuria de 24 horas y el índice proteína/creatinina en pacientes con sospecha de preeclampsia

Mediante la construcción de una curva ROC se obtuvo el mejor punto de corte del índice proteína/creatinina para la población en estudio, siendo este valor igual o mayor a 0,39 , con una $\mathrm{S}$ de 68,3\% (IC $95 \%$ : 60,275,5), E 73,5 \% (IC $95 \%$ : 56,9-85,4), VPP 91,3\% (IC $95 \%$ : 84,4-95,4), VPN 36,2 \% (IC $95 \%$ : 25,9-48), LHR(+) 2,58 (IC $95 \%$ : 1,46-4,57), LHR (-) 0,43 (IC $95 \%$ : 0,33-0,56) y un área bajo la curva de 0,7799 (buena prueba) (Gráfico 2).

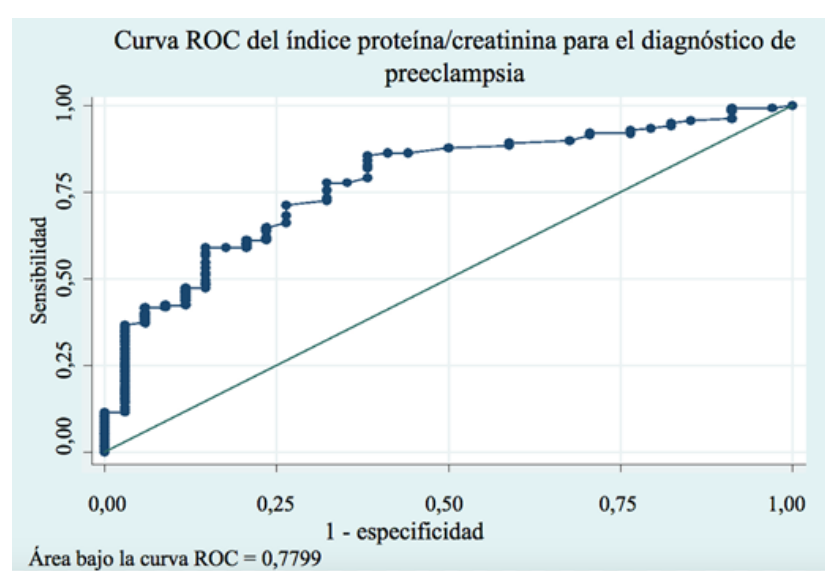

Gráfico 2. Curva ROC del índice proteína/creatinina para el diagnóstico de preeclampsia

Considerando este nuevo punto de corte, 95 pacientes $(54,9 \%)$ tuvieron preeclampsia, mientras que 25 pacientes $(14,45 \%)$ con un índice $<0,39$ no tuvieron preeclampsia. Se compararon las propiedades discriminativas de los puntos de corte del índice proteína/creatinina ( $\geq 0,30 \mathrm{y} \geq 0,39)$ en las tablas 4 y 5 .

Por último al evaluar el área bajo la curva ROC del índice proteína/creatinina y la proteinuria de 24 horas, 0,7799 y 0,8185 , respectivamente, no se encontraron diferencias significativas $(\mathrm{p}=0,0814)($ Gráfico 3$)$

Tabla 4. Comparación de los puntos de corte, convencional y nuevo, del índice $\mathrm{P} / \mathrm{C}$ respecto al diagnóstico de preeclampsia.

\begin{tabular}{|c|c|c|c|c|}
\hline \multirow{3}{*}{ Diagnóstico } & \multicolumn{4}{|c|}{ Índice proteína/creatinina $\mathrm{n}(\%)$} \\
\hline & \multicolumn{2}{|c|}{ Resultado negativo } & \multicolumn{2}{|c|}{ Resultado positivo } \\
\hline & $<0,30$ & $<0,39$ & $\geq 0,30$ & $\geq 0,39$ \\
\hline No preeclampsia & $\begin{array}{c}21 \\
(12,13)\end{array}$ & $\begin{array}{c}25 \\
(14,45)\end{array}$ & $\begin{array}{c}13 \\
(7,51)\end{array}$ & $\begin{array}{c}9 \\
(5,2)\end{array}$ \\
\hline Preeclampsia & $\begin{array}{c}24 \\
(13,87)\end{array}$ & $\begin{array}{c}44 \\
(25,43)\end{array}$ & $\begin{array}{c}115 \\
(66,47)\end{array}$ & $\begin{array}{c}95 \\
(54,91)\end{array}$ \\
\hline
\end{tabular}


Tabla 5. Comparación de las propiedades discriminativas del Índice Proteína/Creatinina $\mathrm{y}$ proteinuria de $24 \mathrm{~h}$

\begin{tabular}{lcc}
\hline \multirow{2}{*}{ Propiedad } & \multicolumn{2}{c}{ Punto de corte del } \\
& 0,30 & 0,39 \\
\hline Índice Proteína/Creatinina \\
Espensibilidad & $82,7 \%$ & $68,3 \%$ \\
VPP & $61,8 \%$ & $73,5 \%$ \\
VPN & $89,8 \%$ & $91,3 \%$ \\
LHR (+) & $46,7 \%$ & $36,2 \%$ \\
LHR (-) & 2,16 & 2,58 \\
Falsos negativos & 0,28 & 0,43 \\
Falsos positivos & $17,3 \%$ & $31,7 \%$ \\
\hline
\end{tabular}

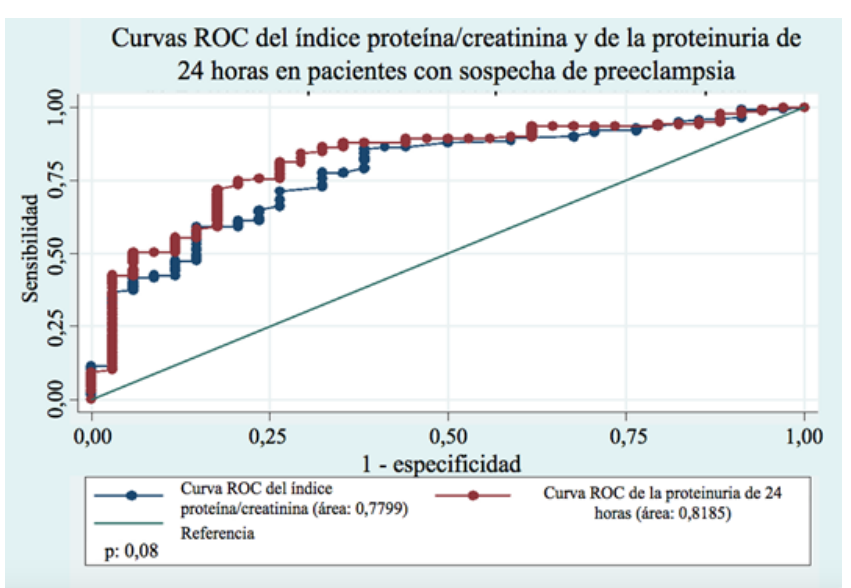

Gráfico 3. Comparación de curvas ROC del índice proteína/creatinina y la proteinuria de 24 horas

\section{DISCUSIÓN}

Las características clínicas y demográficas de la población en estudio difieren a lo reportado en otros trabajos $(25$ - 31). Las pacientes eran de mayor edad gestacional (37,6 vs. 24 - 33), con poca fiabilidad para el cálculo de la misma, presentaban menor número de pacientes primigestas (59 \% vs. $30 \%-34 \%$ ) y pacientes nulíparas ( $45 \%$ vs. $28 \%$ - $34 \%$ ) y menor número de controles prenatales ( $40 \%$ con menos de 6 ) (25 - 31). Sin embargo, los síntomas descritos fueron similares a lo anteriormente reportado, encontrándose solo diferencia significativa en la frecuencia de epigastralgia, la cual fue mayor en el presente estudio (14\% vs. $8 \%$ ) (27). Esta diferencia es importante debido a que dichos trabajos han demostrado que la epigastralgia es un predictor de gravedad y desarrollo de complicaciones en preeclampsia y podría indicar un cuadro más severo en este grupo de pacientes. Lo anteriormente descrito podría atribuirse a un menor nivel de educación, socioeconómico y cultural, así como al pobre acceso a los servicios de salud, y por ende un diagnóstico más tardío $(28,29)$.

El porcentaje de pacientes diagnosticadas con preeclampsia fue mayor utilizando los recientes criterios recomendados por el ACOG en el 2013 (5), en comparación a los del consenso de 2002 (32) ( $80,3 \%$ vs. $64,7 \%$ ). Esto se explicaría porque los nuevos criterios hacen énfasis en la presencia de hipertensión arterial asociada a signos y síntomas de órganos blancos y no consideran indispensable la presencia de proteinuria para el diagnóstico de preeclampsia. Este cambio se debe a que algunas pacientes pueden desarrollar y/o manifestar compromiso de otros órganos antes de que ocurra en el riñón con la manifestación de proteinuria (33 - 35).

Anteriormente se consideraba solo la proteinuria de 24 horas y, en algunos casos, la tira reactiva, para la detección de proteinuria significativa (32). Estudios previos han demostrado sin embargo que el tiempo que demanda la recolección de orina de 24 horas retrasa el diagnóstico de preeclampsia $(7$ - 15). Los criterios diagnósticos del ACOG (5) incluyen para el análisis de proteinuria significativa al índice proteína/creatinina, dado que por su rápido análisis y aplicación genera un mayor porcentaje de 


\section{DESEMPEÑO DEL ÍNDICE PROTEÍNA/CREATININA PARA PREDECIR PROTEINURIA SIGNIFICATIVA EN PACIENTES CON SOSPECHA DE PREECLAMPSIA}

diagnósticos tempranos de proteinuria significativa y preeclampsia. La celeridad en el diagnóstico generaría un impacto importante en las tasas de morbimortalidad maternofetal $(10-19)$.

Por otro lado, el diagnóstico de preeclampsia grave, ocurrió en menos de la mitad de los casos $(25,4 \%)$ con los nuevos criterios (5), comparado con los diagnósticos que se hubieran hecho con los del consenso de 2002 (32) $(53,7 \%)$. Es probable que el sobrediagnóstico de preeclampsia grave, pueda atribuirse al uso de proteinuria $>$ a $5 \mathrm{~g}$ en 24 horas y a la presencia de retraso de crecimiento intrauterino, criterios que ya no se consideran signos de gravedad para preeclampsia. Estos dos criterios no han demostrado una relación significativa con resultados adversos maternos y/o fetales (33-35). El sobrediagnóstico de preeclampsia grave genera un incremento en la tasa de cesáreas, el uso innecesario de fármacos indicados en los cuadros graves, mayor tiempo de hospitalización, ingresos a la unidad de cuidados intensivos, infecciones intrahospitalarias, prematuridad, bajo peso al nacer, afección psicológica de la paciente y en sus familiares, costes y recursos de salud $(5,32)$.

De las complicaciones registradas, si bien solo 3 pacientes desarrollaron síndrome de HELLP, fueron más las que presentaron daño de órgano blanco; 17 tuvieron alteración hepática, 4 alteración hematológica y 4 alteración renal. Estos resultados son menores a los reportados en un análisis secundario realizado por la Organización Mundial de la Salud en el año 2013 (36), donde se encontró que de las pacientes que tuvieron preeclampsia, $43 \%$ presentaron disfunción hematológica, $24 \%$ disfunción hepática y $16 \%$ disfunción renal. Esta diferencia podría deberse a que en dicho estudio se emplearon los criterios diagnósticos previos de preeclampsia, donde no se incluía al índice proteina/creatinina lo que generaba un diagnóstico más tardío y mayor frecuencia de complicaciones (36). Se puede inferir que con su uso se logra un mayor porcentaje de diagnósticos tempranos, lo que evitaría la progresión de las complicaciones.

La indicación de cesárea en pacientes con preeclampsia varía ampliamente en la literatura (32\% - $60 \%)(25,36)$. Del total de pacientes que terminaron la gestación durante su hospitalización, $75 \%$ fueron por cesárea. Se puede considerar que en el $60 \%$, al no tener el antecedente de cesárea previa, la indicación primaria fue la presencia de preeclampsia y sus complicaciones. Sin embargo, no se puede excluir que en esta decisión puedan haber intervenido además otros factores que se mencionan en la literatura, como pueden ser el temor a los problemas médico legales y la comodidad de culminar rápidamente con la situación de incertidumbre generada por la hipertensión en el embarazo.

Llama la atención que $10 \%$ de las pacientes del estudio fueron dadas de alta sin culminar la gestación, por lo que se hospitalizaron únicamente para el descarte de preeclampsia, generando un gasto innecesario en recursos de salud, lo que pudo haberse evitado con un test de diagnóstico temprano para la detección de proteinuria significativa.

La aplicación de este test puede tener impacto en la reducción de cesáreas y el número de hospitalizaciones. Sin embargo, en este estudio no hubo diferencias en el número de cesáreas con la aplicación del índice proteína/creatinina utilizando el punto de corte convencional versus el nuevo propuesto en este estudio.

Según un metaanálisis publicado en 2012 (11), donde se incluyeron 13 estudios, el punto de corte del índice proteína/creatinina presentaba un amplio rango $(0,15-0,50)$, con sensibilidad entre $0,65 \%$ y $0,89 \%$ y especificidad entre $0,63 \%$ y $0,87 \%$, concluyendo que no existía suficiente evidencia para determinar 
un único punto de corte estándar debido a la heterogeneidad de métodos para el cálculo de la proteinuria y las diferentes características de las poblaciones estudiadas. Esto justifica el objetivo del presente estudio, observando que el índice proteína/creatinina propuesto se encuentra dentro del rango reportado en la literatura y podría ser más específico y sensible y por tanto útil para la población de país.

Al comparar las propiedades discriminativas de los puntos de corte estándar y el calculado $(0,30$ vs 0,39 ), se observa que si bien la sensibilidad disminuye con el nuevo punto de corte $(82,7 \%$ vs $68,3 \%)$, la especificidad aumenta $(67,8 \%$ vs $73,5 \%$ ) (5). Por ser la preeclampsia una patología con alta morbimortalidad, se requiere un punto de corte altamente sensible que ayude a detectar el mayor número de casos posibles, por lo que sería más útil utilizar el punto de corte convencional en el manejo de pacientes en emergencia y en casos de alta sospecha, y reducir así la tasa de morbimortalidad maternofetal. Sin embargo, para el manejo ambulatorio se propone utilizar un test más específico para un mejor descarte de preeclampsia en pacientes con baja probabilidad de desarrollar la misma. El punto de corte hallado en este estudio $(0,39)$ presenta esta característica respecto al convencional, por lo que su utilidad sería mayor en el manejo ambulatorio de las pacientes gestantes. Los autores quieren recalcar que el buen uso de los puntos de corte, ya sea el convencional o el nuevo, debe estar ligado estrechamente a las manifestaciones clínicas, antecedentes, factores de riesgo, comorbilidades y recursos de salud que se dispongan al momento para el diagnóstico de preeclampsia.

La excelente correlación obtenida entre el índice proteína/creatinina y la proteinuria de 24 horas, (rs: 0,$9308 ; p=0,000$ ), que incluso supera la reportada por muchos estudios (rs: 0,484 - 0,84, $\mathrm{p}<0,01-0,0001$ ), refuerza la recomendación de su uso con la ventaja adicional de su facilidad y rapidez de aplicación (13-16). Sin embargo, debe llamar la atención que a mayor concentración de proteinuria en 24 horas (> $5 \mathrm{~g}$ ), hay una mayor dispersión de datos, mostrando una menor relación lineal entre ambas. Esta dispersión también ha sido descrita en otros estudios, observándose que incluso puede iniciarse con niveles de proteinuria de $1 \mathrm{~g}$ en 24 horas (1018). Por tanto, si bien el índice proteína/creatinina es útil para predecir proteinuria significativa y muestra una excelente correlación con la proteinuria de 24 horas, puede ser poco útil en pacientes con proteinuria masiva y en rango maligno. Si este dato se toma en el contexto de la nueva recomendación del ACOG (5), basta que el índice proteína/ creatinina informe que hay proteinuria para hacer el diagnóstico de preeclampsia en una paciente con hipertensión arterial y el criterio de gravedad recaerá en la presencia de algún otro órgano blanco comprometido.

Adicionalmente, otro dato que apoya el uso del índice proteína/creatinina es el hecho que la comparación del área bajo la curva ROC entre esta prueba y la proteinuria de 24 horas $(0,7799$ y 0,8185$)$ no mostró diferencias significativas $(p=0,0814)$. Con este hallazgo, se puede inferir que el índice proteína/creatinina resulta ser un excelente test diagnóstico, al igual que el estándar de oro (proteinuria de 24 horas) para predecir proteinuria significativa.

En conclusión, el nuevo punto de corte para el índice proteína/creatinina es diferente al convencional, resulta ser más específico $(0,39$ vs. 0,3$)$ y por lo tanto podría ser útil en el manejo ambulatorio de las pacientes con sospecha de preeclampsia. Con este nuevo punto de corte se intenta aumentar la sensibilidad y especificidad del mismo, ajustado a las características intrínsecas de la población. 


\section{DESEMPEÑO DEL ÍNDICE PROTEÍNA/CREATININA PARA PREDECIR PROTEINURIA SIGNIFICATIVA EN PACIENTES CON SOSPECHA DE PREECLAMPSIA}

Las limitaciones del presente trabajo fueron que no se realizó una selección aleatoria de las pacientes participantes, no se estandarizó el procesamiento de las muestras de orina y no se incluyeron suficientes pacientes menores de edad por falta de consentimiento informado, pero los autores consideran que estas limitaciones no invalidan los resultados obtenidos y podrían justificar nuevos estudios para resolver estos cuestionamientos.

Finalmente, se puede proponer un nuevo estudio para determinar si los puntos de corte del índice proteína/ creatinina reportados en este estudio, servirían para predecir complicaciones maternofetales en casos de preeclampsia.

\section{REFERENCIAS}

1. Steegers EA, von Dadelszen P, Duvekot JJ, Pijnenborg R. Pre-eclampsia. Lancet. 2010; 376(9741):631-644. doi: 10.1016/S0140-6736(10)60279-6. Epub 2010 Jul 2. PMID: 20598363.

2. Khan KS, Wojdyla D, Say L, Gülmezoglu AM, Van Look PF. WHO analysis of causes of maternal death: a systematic review. Lancet. 2006; 367(9516):10661074. doi: 10.1016/S0140-6736(06)68397-9. PMID: 16581405.

3. Pacheco J. Preeclampsia/eclampsia: Reto para el ginecoobstetra. Acta med peruana [Internet]. 2006 [consultado febrero 2020]; 23(2):100-111. Disponible en: http://www.scielo.org.pe/scielo.php?script=sci_ arttext\&pid=S1728-59172006000200010

4. Del Carpio L. Situación de la mortalidad materna en el Perú, 2000 - 2012. Rev Peru. Med Exp Salud Pública [Internet]. 2013 [consultado febrero 2020]; 30 (3):461-464. Disponible en: http://www.scielo.org.pe/scielo.php?script $=$ sci arttext\&pid=S1726-46342013000300015

5. American College of Obstetricians and Gynecologists; Task Force on Hypertension in Pregnancy. Hypertension in pregnancy. Report of the American College of Obstetricians and Gynecologists' Task Force on Hypertension in Pregnancy. Obstet Gynecol. 2013; 122(5):11221131. doi: 10.1097/01.AOG.0000437382.03963.88. PMID: 24150027.

Vol. 80, No 4, diciembre 2020.
6. Report of the National High Blood Pressure Education Program Working Group on High Blood Pressure in Pregnancy. Am J Obstet Gynecol. 2000; 183(1):S1-S22. PMID: 10920346.

7. Côté AM, Firoz T, Mattman A, Lam EM, von Dadelszen P, Magee LA. The 24-hour urine collection: gold standard or historical practice? Am J Obstet Gynecol. 2008; 199(6):625.e1-6. doi: 10.1016/j.ajog.2008.06.009. Epub 2008 Aug 21. PMID: 18718568 .

8. Waugh J, Bell SC, Kilby MD, Lambert P, Shennan A, Halligan A. Urine protein estimation in hypertensive pregnancy: which thresholds and laboratory assay best predict clinical outcome? Hypertens Pregnancy. 2005; 24(3):291-302. doi: 10.1080/10641950500281019. PMID: 16263601.

9. Wongkitisophon K, Phupong V, Yamasmit W, Pansin $\mathrm{P}$, Tannirandorn Y, Charoenvidhya D. Correlation of 4- and 24-hour urine protein in women with initially diagnosed hypertensive disorders in pregnancy. J Med Assoc Thai. 2003; 86(6):529-534. PMID: 12924801.

10. Côté AM, Brown MA, Lam E, von Dadelszen P, Firoz T, Liston RM, et al.. Diagnostic accuracy of urinary spot protein:creatinine ratio for proteinuria in hypertensive pregnant women: systematic review. BMJ. 2008; 336(7651):1003-1006. doi: 10.1136/ bmj.39532.543947.BE. Epub 2008 Apr 10. PMID: 18403498; PMCID: PMC2364863.

11. Aggarwal N, Suri V, Soni S, Chopra V, Kohli HS. A prospective comparison of random urine proteincreatinine ratio vs 24-hour urine protein in women with preeclampsia. Medscape J Med. 2008; 10(4):98. PMID: 18504482; PMCID: PMC2390693.

12. Dwyer BK, Gorman M, Carroll IR, Druzin M. Urinalysis vs urine protein-creatinine ratio to predict significant proteinuria in pregnancy. J Perinatol. 2008; 28(7):461-467. doi: 10.1038/jp.2008.4. Epub 2008 Feb 21. PMID: 18288120; PMCID: PMC2743480.

13. Morris RK, Riley RD, Doug M, Deeks JJ, Kilby MD. Diagnostic accuracy of spot urinary protein and albumin to creatinine ratios for detection of significant proteinuria or adverse pregnancy outcome in patients with suspected pre-eclampsia: systematic review and meta-analysis. BMJ. 2012; 345:e4342. doi: 10.1136/ bmj.e4342. PMID: 22777026; PMCID: PMC3392077.

14. Taherian A, Dehbashi S, Baghban M. The Relationship between Random Urinary Protein-to-Creatinine Ratio 
and 24-hours Urine Protein in Diagnosis of Proteinuria in Mild Preeclampsia. JRMS [Internet]. 2006 [consultado febrero 2020]; 11(1): 6-12. Disponible en: https:// www.researchgate.net/publication/41390701_The_ Relationship_between_Random_Urinary_Protein-toCreatinine_Ratio_and_24-hours_Urine_Protein_in Diagnosis_of_Proteinuria_in_Mild_Preeclampsia

15. Mohseni SM, Moez N, Naghizadeh MM, Abbasi M, Khodashenas Z. Correlation of random urinary protein to creatinine ratio in 24-hour urine samples of pregnant women with preeclampsia. J Family Reprod Health. 2013; 7(2):95-101. PMID: 24971109; PMCID: PMC4064777.

16. Shahbazian N, Hosseini-Asl F. A comparison of spot urine protein-creatinine ratio with 24-hour urine protein excretion in women with preeclampsia. Iran J Kidney Dis. 2008; 2(3):127-131. PMID: 19377225.

17. Eslamian L, Behnam F, Tehrani ZF, Jamal A, Marsoosi V. Random urine protein creatinine ratio as a preadmission test in hypertensive pregnancies with urinary protein creatinine ratio. Acta Med Iran. 2011; 49(2):81-84. PMID: 21598214.

18. Yamasmit W, Chaithongwongwatthana S, Charoenvidhya D, Uerpairojkit B, Tolosa J. Random urinary protein-to-creatinine ratio for prediction of significant proteinuria in women with preeclampsia. J Matern Fetal Neonatal Med. 2004; 16(5):275-279. doi: 10.1080/14767050400018056. PMID: 15621543.

19. Sethuram R, Kiran TS, Weerakkody AN. Is the urine spot protein/creatinine ratio a valid diagnostic test for pre-eclampsia? J Obstet Gynaecol. 2011; 31(2):128130. doi: 10.3109/01443615.2010.538771. PMID: 21281026.

20. Vassalotti JA, Stevens LA, Levey AS. Testing for chronic kidney disease: a position statement from the National Kidney Foundation. Am J Kidney Dis. 2007; 50(2):169-180. doi: 10.1053/j.ajkd.2007.06.013. PMID: 17660017.

21. National Kidney Foundation. K/DOQI clinical practice guidelines for chronic kidney disease: evaluation, classification, and stratification. Am J Kidney Dis. 2002; 39(2 Suppl 1):S1-266. PMID: 11904577.

22. Christopher-Stine L, Petri M, Astor BC, Fine D. Urine protein-to-creatinine ratio is a reliable measure of proteinuria in lupus nephritis. J Rheumatol. 2004; 31(8):1557-1559. PMID: 15290735.

23. Torng S, Rigatto C, Rush DN, Nickerson P, Jeffery JR. The urine protein to creatinine ratio $(\mathrm{P} / \mathrm{C})$ as a predictor of 24-hour urine protein excretion in renal transplant patients. Transplantation. 2001; 72(8):1453-1456. doi: 10.1097/00007890-200110270-00021. PMID: 11685120.

24. Waugh JJ, Clark TJ, Divakaran TG, Khan KS, Kilby MD. Accuracy of urinalysis dipstick techniques in predicting significant proteinuria in pregnancy. Obstet Gynecol. 2004; 103(4):769-777. doi: 10.1097/01. AOG.0000118311.18958.63. PMID: 15051572.

25. Kumari A, Chakrawarty A, Singh A, Singh R. Maternofoetal complications and their association with proteinuria in a tertiary care hospital of a developing country. J Pregnancy. 2014; 2014:431837. doi: 10.1155/2014/431837. Epub 2014 Apr 14. PMID: 24829801 ; PMCID: PMC4009128.

26. Akaishi R, Yamada $T$, Morikawa M, Nishida R, Minakami H. Clinical features of isolated gestational proteinuria progressing to pre-eclampsia: retrospective observational study. BMJ Open. 2014; 4(4):e004870. doi: 10.1136/bmjopen-2014-004870. PMID: 24747797; PMCID: PMC3996810.

27. Stout MJ, Scifres CM, Stamilio DM. Diagnostic utility of urine protein-to-creatinine ratio for identifying proteinuria in pregnancy. $\mathrm{J}$ Matern Fetal Neonatal Med. 2013; 26(1):66-70. doi: 10.3109/14767058.2012.727048. Epub 2012 Oct 5. PMID: 23020712; PMCID: PMC4118637.

28. Thangaratinam S, Gallos ID, Meah N, Usman S, Ismail KM, Khan KS; TIPPS (Tests in Prediction of Preeclampsia's Severity) Review Group. How accurate are maternal symptoms in predicting impending complications in women with preeclampsia? A systematic review and meta-analysis. Acta Obstet Gynecol Scand. 2011; 90(6):564-573. doi: 10.1111/j.1600-0412.2011.01111.x. Epub 2011 Apr 15. PMID: 21355860.

29. Bilano VL, Ota E, Ganchimeg T, Mori R, Souza JP. Risk factors of pre-eclampsia/eclampsia and its adverse outcomes in low- and middle-income countries: a WHO secondary analysis. PLoS One [Internet]. 2014 [consultado febrero 2020]; 9(3):e91198. Published 2014 Mar 21. doi:10.1371/journal.pone.0091198

30. Ohuma EO, Papageorghiou AT, Villar J, Altman DG. Estimation of gestational age in early pregnancy from crown-rump length when gestational age range is truncated: the case study of the INTERGROWTH21st Project. BMC Med Res Methodol. 2013; 13:151. doi: 10.1186/1471-2288-13-151. PMID: 24314232; PMCID: PMC4029763.

31. Abou Zahr CL, Wardlaw TM. Antenatal Care in Developing Countries: Promises, Achievements and Missed Opportunities: An Analysis of Trends, Levels 
and Differentials, 1990-2001 [Internet]. Geneva: World Health Organization; 2003. Disponible en: https://www.who.int/reproductivehealth/publications/ maternal_perinatal_health/9241590947/en/

32. ACOG Committee on Obstetric Practice. ACOG practice bulletin. Diagnosis and management of preeclampsia and eclampsia. Number 33, January 2002. American College of Obstetricians and Gynecologists. Int J Gynaecol Obstet. 2002; 77(1):67-75. PMID: 12094777.

33. Homer CS, Brown MA, Mangos G, Davis GK. Non-proteinuric pre-eclampsia: a novel risk indicator in women with gestational hypertension. J Hypertens. 2008; 26(2):295-302. doi: 10.1097/ HJH.0b013e3282fla953. PMID: 18192844.

34. Schiff E, Friedman SA, Kao L, Sibai BM. The importance of urinary protein excretion during conservative management of severe preeclampsia. Am J Obstet Gynecol. 1996; 175(5):1313-1316. doi: 10.1016/s0002-9378(96)70047-9. PMID: 8942507.
35. Newman MG, Robichaux AG, Stedman CM, Jaekle RK, Fontenot MT, Dotson T, et al. Perinatal outcomes in preeclampsia that is complicated by massive proteinuria. Am J Obstet Gynecol. 2003; 188(1):264268. doi: 10.1067/mob.2003.84. PMID: 12548227.

36. Abalos E, Cuesta C, Carroli G, Qureshi Z, Widmer $\mathrm{M}$, Vogel JP, et al. WHO Multicountry Survey on Maternal and Newborn Health Research Network. Pre-eclampsia, eclampsia and adverse maternal and perinatal outcomes: a secondary analysis of the World Health Organization Multicountry Survey on Maternal and Newborn Health. BJOG. 2014; 121 Suppl 1:14-24. doi: 10.1111/1471-0528.12629. PMID: 24641531

Recibido 31 de marzo de 2020 Aprobado 25 de junio de 2020 